\title{
The Impossible Made Real: A Typology of Loops and an Exploration of the Impact of Immediacy and Hypermediacy in Popular Music
}

\author{
Paul Carr
}

University of South Wales

paul.carr@southwales.ac.uk

\section{Ben Challis}

\author{
Manchester Metropolitan University \\ b.challis@mmu.ac.uk
}

\begin{abstract}
All popular music has a degree of repetition at a micro and/or macro level, a paradigm that has also been shown to be true in both the European classical tradition and music of most other cultures. The occurrences of these events can range from the smallest motific melodic fragment, to 'phrase' (question/answer) and 'section' (verse chorus) repetitions, to riff based harmonic/melodic patterns. These repetitions usually occur on an intra compositional basis, but as evidenced by the pervasiveness of sampled loops in contemporary dance music and rap, can also work on inter compositional levels, resulting in potential conceptual allusions of musical (and non-musical) factors between texts. This essay examines the creative incorporation of a specific type of repetition in popular music, that of loop-based composition and improvisation.
\end{abstract}

KEYWORDS: Loops, Hypermediacy, popular music, composition

\section{Introduction}

We would like to open this essay by agreeing with Richard Middleton's perspective that "all popular songs, to a greater or lesser extent, fall under the power of repetition" (Middleton, 2006: 15). This is a viewpoint that has also been shown to be true in both the European classical tradition and the music of many other world cultures. Indeed, repetition in Western music has a long-standing tradition when 
viewed from both macro and micro perspectives, terminology that Middleton describes as discursive and musematic, respectively (Middleton, 2006: 16). For example, the Sonata Form, as employed by Classical period composers, pervasively employs repetition on both levels, with regular melodic recursions being indicative of the former, and repetition of the initial exposition by the recapitulation representing the latter. These repetitions usually occur on an inter-compositional basis, but as evidenced by composers such as Monteverdi and Prokofiev's reuse of their own similar compositional materials between compositions or Charles Ives' regular inclusion and allusion of other composers' work, intra-compositional levels of repetition are not only apparent, but also have the potential to result in a plethora of complex semiotic relationships between texts.

This essay intends to examine the creative incorporation of a specific type of repetition in popular music, that of loop-based composition (and to a lesser extent improvisation). After presenting a definition of a loop, the essay will progress to outline an initial typology of the ways in which loops are used in music, in both conventional performance environments, and more explicitly with the aid of technology. This will be followed by a brief overview of the history of tape and digital based looping, and concluded by a philosophical examination of the means through which technological looping can be conceptualised in modern day practices.

\title{
What is a Loop in Music?
}

First, it is important to verify that the terminology 'loop' can be seen to be used in numerous disciplines such as science and technology (for example an electric circuit), mathematics (for example loop algebra and graph theory), computers (for example the infinite loop), and of course music. While the Cambridge Dictionary usefully describes loops as either a noun (for example the tape-loop) or a verb (For example the process of looping), we would like to spend a short time extending these definitions, with a particular emphasis on music.

When describing one of his early experiments with 'Frippertronics', Robert Fripp describes the following process, which commences with Fripp entering notes into his duel tape recorder setup one morning. Listening to the emotional impact of the repetitions, he comments

\begin{abstract}
About five minutes later [after setting up the loop] I stumbled over and punched in a few more tones, which turned out to be not the ones I wanted, but I let them stand. This "music" went on and on and on, through breakfast and watering the plants and the rest of it, and by half an hour later the sound had come to seem endowed with a shimmering depth of significance. (Tamm, 1989: 46).
\end{abstract}

This perspective is congruent with Fripp's colleague, Brian Eno, who once asserted that "Almost any arbitrary collision of events listened to enough times comes to seem very meaningful" (Tamm 1989: 56). To be sure, this juxtaposition of continuous repetition and consequent significance is one of the factors that this essay attempts to negotiate.

This practice is particularly prevalent in both mainstream popular music and more contemporary forms of classical music, not only on a melodic basis, but also via a variety of textural constructions highlighted below. These examples range from compositions that rely exclusively on loops for the entirety of their duration, 
to others which selectively incorporate them for specific sections. It is important to highlight that many compositions incorporate a number of techniques both diachronically and synchronically, something that will be addressed as the essay progresses. Interestingly, some of these processes are similar to the theory of Organicism - where a musical work is conceptualised as an organism with individual parts combining to form part of a functioning whole and the body acting as a metaphor for the musical work. This concept has its roots in the work of philosopher George Hegel (1770 - 1831), who states that "if the work is a genuine work of art, the more exact the detail the greater the unity of the whole" (Beard \& Gloag, 2005: 94). This essay extrapolates the means through which this absolute focus on detail can be elaborated upon, and linked both empirically and extramusically to the experience of those involved in both the production and reception of music.

After presenting a brief outline of the history of looping technology, what follows is an initial attempt at a typology of loops, as they pertain to music, where, in addition to widely known looping techniques such as ostinati and riffs, we attempt to develop an extended list of loop descriptions. Finally, we will align both formalist and extra-musical qualities to these descriptions.

\section{A Brief History of Technology for Looping}

To appreciate various compositional applications of repetition, it is perhaps only logical that composers should look to new technologies that assist with this fundamental musical process that creates structure through continuously repeating sonic motifs. Historically this has led to the emergence of novel and often experimental devices, the new techniques that are afforded by them and even the vocabulary by which these new techniques and sounds can be described. In a more contemporary perspective, these home-built solutions have paved the way for a mainstream realisation of similar concepts that are available in forms that now suit mass consumption. Perhaps now regarded as a norm in popular music composition, pattern-based approaches for creating and sustaining new repetitive ideas are evident in many, if not most, common software production tools. Patterns and loops can now be layered to create complex sonic experiences with such immediacy that ideas can be developed or abandoned with ease. In considering the state-of-the-art technologies and fully appreciating the opportunities that they afford, it is worth first reflecting on the various historical developments that have occurred along the way.

\section{Locked Groove Recordings}

The earliest examples of technology-enabled audio loops are generally attributed to the experimental musical works of French composer Pierre Schaeffer. Regarded as the founding figure behind the Music Concrète movement, Schaeffer employed acetate disc recordings to capture real or 'concrete' sounds that were manipulated and layered to create rich sonic landscapes. One technique that Schaeffer employed was to interrupt the spiral groove on a recording to create a "closed" or "locked" groove. Unlike the terminating locked-groove that prevented the stylus from progressing onto an album's label, Schaeffer's closed loops contained recorded sound and were also situated at strategic distances on the surface to offer different loop lengths. Clear examples of this technique can be heard within his collection of etudes "Cinq études de bruits" (1948) and perhaps most notably 
"Etude aux chemins de fer" (1948), where various sounds from trains are looped to create mechanical rhythmic patterns. Although locked-grooves of this type were superseded by the possibilities offered by the emergence of magnetic tape, the concept remained in use as a novel ending to many commercial recordings by bands and artists on vinyl recordings from the 1960s onwards. For example, at the end of The Beatles' Sgt. Pepper's Lonely Hearts Club Band album (1967), the closed-groove is used to store random layered voices and the final groove of King Crimson's USA (1975) produces a cycling loop of applause. More recently, Stereolab's final track from Transient Random Noise Bursts With Announcements (1993) enters into a terminal loop at its close with the clue being in the track's title "Lock Groove Lullaby". Additionally, the Super Furry Animals multi-disc album Rings Around the World (2001) features an entire side containing a single closedgroove in the middle of the disc; the groove plays a timed sample of the basic groove from "All the shit $U$ do" (2001), a non-album track.

\section{Magnetic Tape}

Though the concept of looped audio-recordings clearly originates from the early experiments of Schaeffer and his contemporaries, it is not clear whether the term 'loop' as used in a sonic context emerged at this same time. It is more likely that the term was first used in reference to tape-loops. Even if this is the case, it is not clear who first coined the phrase or indeed who first explored the transition of the idea from acetate to magnetic tape. During the early 1950s, experimental composers Louis and Bebe Barron used tape-loops in their works. In an interview with composer Jane Brockman (1992), Bebe Barron suggests that she may well have been the originator of both the phrase and the concept. In response to a question by Brockman as to whether she (Barron) had invented the tape-loop, Barron replied:

I think we did. I never heard of anyone else doing it at the time. In 1949, Stancil-Hoffman offered to make us a tape recorder to our specifications so we took advantage of it, I believe it was the first commercial tape recorder ever made. Bebe Barron (1992).

Yet, it is also clear that Les Paul was experimenting with tape-loops at a similar time, in both his pioneering work with multi track recording techniques, and live performance, via an invention he intitled the 'Pulverisor'. At a similar time, Pierre Schaeffer's interest in sonic loops moved into the use of magnetic tape and ultimately to the commission of a device called the 'Morphophone'. Created by Fances Poullin in the 1950s for Schaeffer, this device featured a revolving cylinder of $50 \mathrm{~cm}$ with a loop of magnetic tape around it. Armed with twelve heads (record, erase and then ten moveable heads for playback) a short recording could be cycled around indefinitely with different timed delays being offered by the spacing of the playback heads (Teruggi 2007). Two paths arguably emerge at this point: one that moved towards the use of closed loops of magnetic tape to produce prolonged echo delays as commercialised within products like the Echoplex (c. 1959) and one that moved towards the use of closed-loops to accumulate layers. Though the use of tape-delay is of interest within the scope of this essay, it is perhaps the latter use of magnetic tape, to emulate the closed-groove concept whilst offering the potential to introduce further layers that is of more relevance.

The most notable early explorer of tape-layering using loops in this way is Terry Riley. Prominent as an experimental composer and performer within the emerging minimalist music movement of the early 1960s, Riley conceived a structural idea 
based on long loops of sound, being layered and adapted over substantial periods of time. Using two reel-to-reel tape recorders, Riley developed the 'time lagaccumulator' for this very purpose, using the device to create huge live performances, creating loops whilst simultaneously improvising over them. His albums Reed Streams (1966) and Rainbow in Curved Air (1969) are indicative of the live performances that Riley achieved at the time. A more mainstream application of the same technique was offered by Robert Fripp and Brian Eno in their 1973 collaboration on No Pussyfooting, with Fripp subsequently coining the term 'Frippertronics' for his interpretation of Riley's original device.

\section{Digital Audio}

Originally conceived as digital counterparts to the earlier tape-based echo machines (Echoplex c. 1959 and WEM Copicat c. 1958), early digital delay pedals such as the Boss DD2 (c. 1984) offered only a few seconds of delay time, but if feedback was set to a high level, this could effectively be turned into a closed-loop effect. In this respect, digital delay pedals could offer similar capabilities for layering to those explored by the likes of Riley and Fripp, but working with much shorter loops. The first dedicated digital loop-recorder was the Paradis Loop Delay (c. 1992), a device where the functionality reflected the use of continuous loops more than it did the use of gradually diminishing echo-like delay. As with the division of paths in the evolution of analogue approaches to delay devices and looping devices, similar is true within the digital domain seeing FX approaches to delay developing in parallel to sustained structural development of continuous loops. The latter leading to the development of dedicated loop stations.

\section{Analogue Step Sequencers}

Although the development of digital means for storing and manipulating new audio possibilities were quickly realised for taking the potential for working with loops to new levels, it is important to acknowledge the evolution of another approach to loop-like generation of patterns in electronic music that was progressing in parallel around this same time.

Companies producing modular synthesisers in the 1960s began to offer modules that would allow a series of control voltages $(\mathrm{CVs})$ to be cycled in a seemingly endless loop. In the most basic of formats, modules such as the Moog 960 step sequencer could send these $\mathrm{CV}$ s to produce, for example, a series of pitched notes, the sequencer would then cycle through this tone-row by a speed set by clock circuit. Although seemingly basic in terms of melodic development, a stepsequencer of this sort was a highly effective method of creating and controlling ostinato patterns. A technique that was used extensively by, for example, Tangerine Dream, became a common feature of less modular, dedicated synthesisers of the type favoured by the electronic bands of the early 1980s, who continued to use the step-sequencer as a generator for laying down ostinato grooves (for example "Dreams of Leaving" (Travelogue 1980) by The Human League). Though more flexible approaches to sequencing (particularly using the MIDI protocol) have emerged in the decades that have followed, it is interesting to note that the concept of step-sequenced loops and/or repeated patterns has remained, frequently being presented as a fundamental building block for composition. 


\section{Phrase Samplers and Hybrid Devices}

The contemporary music composition and production suite is likely to incorporate software environments of the types just mentioned, platforms for fast creation and manipulation of looped phrases along with looped digital audio. These have become the established norm for much of the recorded commercial music that is being produced for today's market. Yet, the experimental interest in loops remains (for example, artists such as Add $\mathrm{N}$ to $(\mathrm{X})$, Aphex Twin, Mùm), as does the desire by performers to improvise with looped material (for example Son of Dave, Imogen Heap) and so the technological development of hardware and software tools for loop-based performance continues. Most recently, the Korg Kaos series, is offering hybrid devices for recording multiple loops and manipulating them in realtime (Kaospad) or for generating and synthesised phrases and recorded loops to similar effect (Kaossilator).

\section{A Proposal for a Taxonomy of Loops in Music}

Personal experience in attempting to articulate the relevance and significance of looped material and its usage within contemporary music suggests that there is scope for a musicological model that specifically considers this type of creative practice. It is also quite apparent that a shared vocabulary or set of defining terms and labels is still to emerge from within the discipline. The model being presented here is still in its infancy and is not intended to be definitive. However, the anticipation is that the suggestion of a plausible model and the ensuing dialogue that this can initiate will contribute towards an on-going development of a more coherent, and ultimately, more complete structure.

\section{Overview}

The model is essentially a tool for referencing the collective salient features contained within a looped extract or within its context that afford it some level of individuality or identity. There are three levels of consideration within the process of identification: a base Type, any number of Formalist Qualities and, finally, ExtraMusical Qualities.

\section{Type}

Defining a Type for a given loop is perhaps similar to considering its place within a group of discrete categories or families. However, it is also acknowledged that a loop may exist quite comfortably within more than one of these base level groupings such that its initial property will be to have one or more Types. At present, six potential descriptors are proposed.

1. Multipart: containing multiple instrument parts. This Type is an attribute of much popular music, which has long featured multiple instruments (for example drum set, bass, guitar, keys, etc.) performing individual loops, often for specific sections of a composition. Indicative examples range from Elvis Presley's early recordings such as "Hound Dog" (1957) and "All Shook Up" (1957), to funk tracks such as James Brown's "Get Up Offa That Thing" (1976), to more contemporary 'classical' examples such as Terry Riley's In $C$ (1968) and the introduction of Mike Oldfield's Tubular Bells (1973). It is also noteworthy that modern day popular music 
ensembles such as Foals use polyphonic looping techniques that appear to be a confluence of minimalism and rock music, with compositions such as "Cassius" (Antidotes, 2008) fusing multiple looped guitars, with repetitive bass, drum and vocal patterns.

2. Riff: a single repetitive part that can be melodic or harmonic in nature, forming either the entirety, or a specific section of a piece. Building on ostinato (see below), this type has been a feature of both Jazz and Rock music since the 1920s, although according to Schuller, was used by earlier brass bands to describe repeating refrain structures (1968: 28). Indicative melodic examples in early Jazz range Louis Armstrong's "Yes, I'm In the Barrel" (1925), to Count Basie's "One O' Clock Jump" (1937), to practices that continued through the Bebop period and beyond. Indicative early harmonic and melodic examples in Rock music include The Kinks' "You Really Got Me" (1964) and The Rolling Stones' "(I Can't Get No) Satisfaction" (1965). These techniques have become the corner stone of rock music.

3. Ostinato: although there will be apparent similarities between a repeated riff and an ostinato pattern in a traditional musical sense, for the purposes of this model ostinato is used to suggest an underlying rhythmic property (possibly within a melodic line) that is identifiably constant and even, and used as a foundation for new ideas to develop. By way of example, the introductory section to "Dreams of Leaving" by The Human League (Travelogue, 1980) is constructed from an evenly pulsed series of pitches created using a step-sequencer. A more simplistic ostinato loop approach still is adopted in both "O Superman" (Laurie Anderson, 1981) and "Requiem" (Killing Joke, 1980). Both tracks are built upon a backdrop of an insistently repeating and evenly spaced single note, which forms the basis of subsequent creativity.

4. Homophonic: a cyclic chord progression. This Type essentially identifies an underlying block harmonic movement of the sort prevalent in popular music, with indicative examples ranging from "Wonderful World" by Sam Cooke (1960), to "Bleeding Love" by Leona Lewis (Spirit, 2007). However, it is also acknowledged that homophonic movement can be riff-like in nature, with pieces such as "Jumpin' At The Woodside" (1938) by Count Basie being an indicative example of this practice. As described earlier, more than one Type can be used to signify the fundamental properties of a loop, and in specific instances terminology such as 'homophonic-riff' could be adopted.

5. Drone: a constant sonic texture. Though constant and void of obvious rhythm, the drone must still repeat such that it can be perceived as a loop. A clear example of such a drone can be heard throughout the entirety of "Particles \& Waves" by Cranes (Particles \& Waves, 2004) where an unchanging harmonic texture is created with a guitar. Another example is "Heavenly Music Corporation" by Robert Fripp and Brian Eno (No Pussyfooting, 1973), where a drone background is recorded by the guitar, as a foundation for improvised overdubs. Though the loop's repetition is apparent in both instances, they do not suggest any additional rhythmic properties beyond the pace of the loop itself. 
6. Abstract: free from 'formal' musical constraints, such that there is no sense of tonal or rhythmic structure beyond that suggested by the repetition of the loop itself. Beyond the use of atonal pitch-sets, an abstract loop might be derived from environmental sounds (e.g.) or perhaps synthetic 'noise'. This was identified earlier as a technique that was first pioneered in the tape manipulations of the Music Concrète movement; an analogue technique that continues to be explored within much contemporary music but through digital means.

\section{Formalist Qualities}

Once the base type(s) has been established, any number of Formalist Qualities can be attached to further define a given loop's identity. These qualities concern the actual physical properties of the music itself, and presently, the following set of qualities are proposed.

1. Sourced: borrowed or sampled from the work of another artist. Since the advent of sampling, this quality has been pervasive in popular music, although after the Grand Upright Music, Ltd vs Warner Bros ruling of 1991, there was a marked limitation in the capacity of artists to incorporate samples into their work. An indicative example is "December 4th" by Jay-Z (The Black Album, 2003), it being apparent that the whole song is based on the introduction of "That's How Long" by The Chi-Lites (1974). Although the Jay Z piece is in verse - chorus form, both sections are based on pitch-shifted looped samples of the Chi-Lites' earlier work that essentially provide a background for additional instrumentation and of course, a rap vocal line. Along similar lines, but within a context of R\&B, the Sugababes' (2001) reworking of "Freak Like Me" by Adina Howard (1995) features extensive use of sampled backing loops taken from Gary Numan's "Are Friends Electric?" (1979). Conceivably closer to a mash-up than a cover-version, "Freak Like Me" draws heavily on three separate sampled passages taken from Numan's original track such that there is a particularly strong feeling of identity retained from the sourced material throughout.

2. Improvised: much of the recorded work of Son of Dave features live improvised looping of this type, enabling him to feature a combination of vocal sounds, harmonica riffs, hand percussion and foot stamping within his work. As his music progresses through time, individual layers are added to create complex cyclic grooves, which on first listening challenge the parameters normally associated with solo performance. It is important to note that improvised within the context of the proposed typology of loops also suggests live or belonging to the moment. Previously recorded improvised material effectively becomes composed and, therefore, original as described in the following section.

3. Original: unsourced composed material. Though there are many examples of material that is sourced, it is also commonplace for composed or improvised original material to be sampled as a loop.

4. Bare: presented with no additional material. This formal quality is a common feature in music and soundscapes for computer games where memory and storage are often a constraint. In effect, complete and 
potentially quite long musical passages are repeated indefinitely during game play. By way of example, the Nintendo DS port of "Syberia" (2008) from games company Microids features an in-game looped edit of the theme from the PC version of the game. Though the original is a rich orchestral work with considerable variation, the DS edit is of a fixed-loop nature where the repetition is particularly noticeable.

5. Backing: presented as a backdrop to additional material. Though any loop will ultimately be perceived as contributing to an overall whole, loops with the formalist quality backing are likely to be multipart. An indicative example can be found in Gavin Bryars' "Sub Rosa" (1986), which is largely based on a repeated section of Bill Frisell's "Throughout" (In Line, 1983).

6. Rhythmic: containing noticeably strong rhythmical properties. For example a drum or percussion loop, or environmental or synthetic 'noise' being used rhythmically. The Chemical Brothers' use of electronic glitches to create a pseudo-percussive loop in "Bahnhof Rumble" (Hanna, 2011) represents and indicative example of this practice.

7. Arhythmic: in conflict with meter, tempo and/or surrounding rhythmic figures. Similarly to the use of melodic and atonal to differentiate between pitch-based ideas being in or out of key, rhythmic and arhythmic can be used to differentiate between seemingly rhythmic loops that do and do not interlock with the surrounding material; indeed, the majority of drum loops will simply be rhythmic. However, in Tunng's "Tale From Black" (Mother's Daughter and other Songs, 2005) the scratch and bump from a record deck stylus is used to create a slow a near rhythmic figure but the period of the loop prevents the figure from tying into the emerging sense of meter.

8. Unison: the same rhythmic/melodic figure is being sounded in another loop, possibly at a different octave (if pitched).

9. Layered: the loop is one of a number of independent layers. This is typical of much live, improvised looping (e.g. Son of Dave, Imogen Heap, Andre LaFosse) where the performer effectively pieces together a backing texture one layer at a time. Though there may be a sense of phrase length in play somewhere within the overall effect, it is quite conceivable that individual layers will be of different lengths such that polyrhythms emerge, a technique that is applied frequently within the work of LaFosse in particular.

10. Contextually placed: where the loop is perceptually repositioned by the surrounding music. This occurs quite frequently and organically in music built with loops containing pitches or harmonies. As a chord progression is altered beneath, or superimposed upon, any given loop the pitches will change in tonal colour. Examples can be heard in Broadcast's "Chord Simple" (The Future Crayon, 2006) where a looped melodic riff is harmonically repositioned by the changes in the bass and also in Lali Puna's "Faking the Books" (Faking The Books, 2004) where a single pitched ostinato loop, layered with a melodic riff are both harmonically repositioned by a synth bass. The technique was also common during the swing period of Jazz, with melody lines such as "C Jam Blues" (Duke 
Ellington, 1943) and "Jumpin' at the Woodside" (Count Basie, 1938) comprising entirely of a looped theme over changing chords.

11. Processed: the loop has been processed to achieve a significant melodic or textual difference (e.g. pitch-shifted, time-stretched, reversed, granulated). When asked about how this technique is incorporated in his music, Andre Lafosse (email communication, 2011) regarded it as a means through which he can "chop up and randomize [his] playing in such a way that it gets spat back at [him] in different and somewhat unpredictable way[s]. When used in this way, the particular quality has the capacity to facilitate creativity in live performance.

The set of Formalist Qualities as just proposed are not yet intended to be seen as exhaustive, rather as a set of those that have so far been identified as being meaningful. Indeed, it is quite conceivable that additional Formalist Qualities will emerge that address the nature of tonal and rhythmic change across the lifespan of an individual loop-cycle (e.g. dynamic change, tonal change, temporal change). Similarly, it is conceivable that loops might be atonal in relation to the harmonic content against which they are superimposed. It is still unclear how significant such qualities might be but it is anticipated that continued application of the model for analytical means will help to establish this further.

\section{Extra-Musical Qualities}

The same returns not, save to bring the different - William James

As noted by academics such as McClary (2006), Auslander (2008) and Zak (2001), electronic modes of production often aim to precipitate immediacy in the listener, becoming noticeable only when closely scrutinising the text. This is congruent to the proposition presented by Bolter and Grusin (1999), who argued that modern society is driven by a desire for realism, a need which is met by a variety of new media forms ranging from spatial innovations like stereo, quadraphonic and threedimensional movies, to the improved digital quality of compact disks and high definition TV. According to Bolter and Grusin, the irony of this process is that in the quest for realism, the technology making this possible is often fore-grounded, resulting in a process they entitle "Hypermediacy", where the audience is reminded of the technological medium, resulting in an increased awareness of seeing (or in music's case, hearing). When considering the work of loop based musicians such as Les Paul, Robert Fripp and Son of Dave, who use technology as a means of generating loops during live performance, from an audience perspective they can be seen to often straddle the divide between the immediacy of more conventional performance and a process we describe as non-realistic hypermediacy, where the technology is perceived not as a means of generating realism, but as a means of creating sounds and textures that often appear to be beyond the scope of what audiences can perceive - the impossible made real! For example, when examining an indicative live performance by vocalist Amy X Neuburg, the combination of the voice, signal processers, and the resultant sound arguably encourages the listener to consider how the link between the voice and the timbres produced are generated. How can a single performer generate these sounds, and why do they not sound like a single performer? Susan McClary's observation that "the closer we get to the source, the more distant becomes the imagined ideal of unmediated presence" (2005: 167) is noteworthy on this occasion, as it could be argued that 
performances such as this display the reality of what is hidden in much commercial pop music - the incorporation of technology. Additionally, McClary's observation also provides an interesting addition to Organicism outlined earlier, with both philosophies indicating that the detail of the individual parts reflect the truth of the whole. In Organicism's case the formal interrelation of the parts is primary, in McClary's, it is the means through which the sounds are achieved. When bridging this ontological gap, Nattiez (1990) provides a useful model which negotiates what he describes as the poietic (the process of how the music is constructed), the immanent (the material reality of the music) and the esthesic (how the music is interpreted by listeners and musicians). When acknowledging that the means of construction and its subsequent reception "do not necessarily correspond" (ibid: 17), Nattiez's perspective resonates with the work of Michel Foucault (Faubion 1999) and Roland Barthes (1977), both of whom questioned the authenticity and authority of the author when interpreting a text. The importance of the listeners' input into the esthesic process is also outlined by Douglas Hofstadter (1980), who describes the autonomy of the listener to interpret a text as follows:

When a system of "meaningless" symbols has patterns in it that accurately track, or mirror various phenomena in the world, then that tracking or mirroring imbues the symbols with some degree of meaning - indeed, such tracking or mirroring is no less and no more than what meaning is (Hofstadter, 1980: xxi).

This not only complies with the perspective outlined by Brian Eno earlier in this essay, but also Kierkegaard's belief that repetition affords "the present and future [to] contend with each other to find an external expression" (Kierkegaard, 1957: 137), continuing "the very fact that it has been makes the repetition into something new" (Ibid: 189).

It is proposed that when analysing loop-based compositions generated via sound processors, it is important to focus on not only the formalistic melodic, harmonic and rhythmic conventions of western music (what Nattiez describes as "immanent", and described in our model as Formalist Qualities), but also, on factors such as the impact of the technology on the performer's creative decision making (What Nattiez describes as "poietic"), in addition to how the music and its performance is received by listeners (What Nattiez describes as "Esthesic"). Our typology proposes to describe these qualities as Extra-Musical, and they raise a number of important questions, such as: how does the performer creatively engage with a loop generating machine from an improvisational and compositional perspective? Exactly how are specific sounds and textures produced in the interface between the loop performer and technology? How does the listener make sense of what appears on the surface to be disjuncture between performer and sound, input and output? And how and why do listeners make meaning out of what can initially be a series of random events?

\section{Conclusions and Indicative Example of the Model in Practice}

As outlined in the brief analysis of the Amy X Neuburg video above, non-realistic hypermediacy can be considered as the means through which sounds and textures emerge in live performance that appear to be beyond what is physically possible for the musician. Although this technological allusion is also prevalent with nonlooped music, the constant repetition of specific musical and textual phrases, in addition to the musicians' physical manipulation of the technology have the impact 
of encouraging the listener to question the viability of what they are hearing, by reminding them of the impossibility they are witnessing. This ranges from simple looping in performances such as Wolfgang Muthspiel and Brian Blade Friendly Travellers Live (2008), in which the technology is used as a fairly obvious backdrop to provide an ostinato for compositional structure and improvisation, to more obscure recordings by Andre LaFosse, who is known as a loop guitarist, and uses the technology as a specific form of creativity. When listening to individual tracks in LaFosse's Normalized (2003) as an indicative example of a solo artist using looping technology, it is apparent that the composer is capable of building Types from our model such as Multipart looped textures, Homophonic cyclic chord progressions and harmonic and melodic background Riffs, in addition to single and Multipart-Drones (for example, the start of "The Proposition"). These multifaceted Types, form the foundation of a range of Formalist Qualities, such as as Rhythmic, Arhythmic, Improvised, Original, Layered and Contextually Placed.

LaFosse's compositions are often quantized into 4/4 time and use short phrase loops that interact in a similar fashion to the drum, bass, guitar, vocal combination of a rock band, and like Laurie Anderson, Amy X Neuburg and Son of Dave, can be regarded as an example of a solo artist who uses loop technology to facilitate and extend the possibilities of solo performance. As opposed to resonating with Roland Barthes' view that "The bastard form of mass culture is a humiliated repetition", (continuing "always new books, new programmes, new films, news items, but always the same meaning") (1975: 24), LaFosse uses technology to incorporate the minimalist mantra "repetition as a form of change", made famous by Brain Eno (Tamm 1989: 25). Although live recorded performances by LaFosse are not common place, what is available clearly encapsulates our notion of nonrealistic hypermediacy, with the looped sounds produced being at a disjuncture from that of a normal solo guitar performance. As this particular live performance consists almost exclusively of looped material, the constant repetition of its constituent parts, paired with LaFosse's manipulation of effects pedals, arguably encourages (some) listeners to focus not only on repetitive Types (such as multipart, riffs drones, and abstract) and Formalist Qualities (such as original, improvised, rhythmic, and melodic), but also the means through which this impossibility occurs. When asked if he gained any satisfaction from watching an audience attempt to figure out what he was doing, LaFosse commented:

Yes and no - to be honest, I think it's an all-too-common trap for looping
musicians to rely on the supposed 'novelty' of what they're doing to generate
interest for the audience. It's fine to geek out over the technical minutiae of a
performance, and I'm certainly a big enough geek to be very into that
aspect. But my primary goal is for the performance itself to convey all of the
necessary interest to hold up as a performance. If there's a bonus added value
for the complexity of the technique, that's fine, but it's important to me that the
technique be in service of the musical statement. (Email correspondence 2011)

As outlined in our Formalist Qualities, looping can be seen to include Improvised and Original choices for the musician, with the dividing line between these factors often being difficult to decipher for the listener. LaFosse outlined his use of these qualities as follows

Most of my looping exists somewhere on the continuum between totally free improvisation and totally pre-arranged, through-composed material. Because repetition is a very powerful way of creating a sense of structure and meaning, 
the extent to which something is composed vs. improvised can be constantly in flux. There are some 'tunes' I can plug in to the looping process, and there are some strategies I have for taking something random and developing it in a 'theme and variation' sense as well. For me, the ratio of composed-vsimprovised is constantly in flux. (ibid)

For LaFosse, the "flux" of the composed-improvised continuum is seen as part of the poietic impulse, and in many ways his comment reiterates Hofstadter's comment above concerning how repetition can make meaning out of otherwise "meaningless" symbols.

Despite that range of techniques used by LaFosse, as his use of looping technology incorporates principally Original and Improvised Formal Qualities, it arguably lacks the esthesic dimensions of Sourced Formal Qualities outlined in our typology. In many respects, looping (which by default accentuates) sourced material makes it problematic to disguise the copyright clearance issues discussed earlier that have emerged in the last twenty years, but providing copyright clearance can be obtained, we propose that the constant repetition of recorded material has a more profound semiotic impact than an isolated one shot. By listening to the earlier Jay-Z example "December 4th" (The Black Album, 2003), it is apparent that Jay-Z has not only used a sped-up version of the original track, but has used selected loops of the original love song as a means of precipitating an autobiographical discussion about his life as a young person. This dialogue often uses uncompromising language, and directly contrasts with the overt sentimentality of the original track, therefore potentially conflicting with the original artists' poietic intent. As it is a legal right of a composer to contest the adaptation of their work by others, it has become a highly subversive form of expression, and although the law has attempted to come to terms with this process (via the Grand Upright Music, Ltd vs Warner Bros ruling of 1991), as indicated in the Mash-Up mixes of albums such as Danger Mouse's The Grey Album (2004), sampling, and looping in particular can still be rebellious. This album is a fusion of The Beatles The White Album (1968) and (ironically) Jay-Z's The Black Album (2003), and as depicted in a promotional video, looping techniques accentuate the "Oh Yeah" of The Beatles' "Glass Onion" (The White Album, 1968) in a particular obvious anti-authoritarian gesture.

Although musicologists such as Hanslick (1854) have argued against any metamusical meaning being inherent in a text, it is proposed that the combination of specific songs, repetition and recorded sound have numerous possibilities for assisting extra-musical meaning. Recorded sound is inextricably linked to not only specific artists, but also the specific times and places that the artist worked in, so although the relationship between the artist's poietic intent and audiences interpretation is by no means guaranteed, when these constituent parts of a recording are combined with repetition, the extra-musical, or what Richard Middleton would describe as secondary meaning (1990: 220), has an opportunity to flourish. Like the ways in which modern day sampling techniques allow musicians to build upon and reconfigure previously recorded music (legally and otherwise), this essay has hopefully provided a framework for other academics to debate and, of course, develop. Although not intended as a definitive typology, it is a starting point to precipitate dialog in this fascinating subject area. 


\section{Acknowledgements}

This article was originally published as a book chapter in French as Quand l'impossible devient réel : typologie des boucles, impact de l'immédiat et de I'hypermédia sur la pop music ("The Impossible Made Real: A Typology of Loops and an exploration of the impact of immediacy and hypermediacy in Popular Music"). See Livo Bettoi, Michel Delville, Christophe Levaux, Christophe Pirenne, Boucle et Répétition: musique, littérature, arts visuels, 2012. Collection Clinamen, pp. 85-102.

\section{References}

\section{Bibliography}

Auslander, P. 2008. Liveness. London: Routledge.

Barthes, R. -

1975. The Pleasure of the Text. New York: Farrar, Straus and Giroux.

1977. Image Music Text. London: Fontana Press.

Beard, D. and Gloag, K. 2005. Musicology: The Key Concepts. London: Routledge

Bolter, J. and Grusin, D. 2000. Remediation: Understanding New Media. Massachusetts: MIT Press.

Brockman, J. 1992. The First Electronic Filmscore - Forbidden Planet: A Conversation with Bebe Barron, in The Score: The Society of Composers and Lyricists, VII (3), pp. 5, 12-13.

Foucault, M. in Faubion J. (ed.) (1999), 'What Is An Author?', in Aesthetics, Method, And Epistemology: Essential Works of Foucault, 1954-1984. The New Press New York, pp. 205-222.

Hanslick, E. 1986(1854). On the Musically Beautiful. Indiana: Hackett Publishing Company.

Hofstadter, D. 1980. Gödel, Escher, Bach: An Eternal Golden Braid. London: Penguin Books.

Kierkegaard, S. 1957. The Concept of Dread. Translated by Walter Lowrie. Princeton: Princeton University Press.

Nattiez, J-J. 1990. Music and Discourse: Towards a Semiology of Music. New Jersey: Princeton University Press.

Schuller, G. 1968. Early Jazz: Its Roots and Early Development. Oxford: Oxford University Press

Tamm, E. 1989. Brian Eno: His Music and the Vertical Color of Sound. Massachusetts: Da Capo Press.

Teruggi, D. 2007. "Technology and Musique Concrete: The Technical Developments of the Groupe de Recherches Musicales and Their Implication in Musical Composition", Organised Sound 12 (3) pp. 213-31.

McClary, S. 2006. "This Is Not A Story My People Tell", in Bennett, A., Shank, B. and Toynbee, J. The Popular Music Studies Reader. Milton Park: Routledge, pp. 21-28.

Middleton, R. -

1990. Studying Popular Music. Buckingham: Open University Press. 
2006. 'In the Groove or Blowing Your Mind: The Pleasures of Musical Repetition', in Andy Bennett, Barry Shank, Jason Toynbee (Eds.), The Popular Music Studies Reader London: Routledge. pp.15-20

Zak, A. 2001. The Poetics of Rock. Berkeley: University of California Press.

\section{Discography}

Anderson, Laurie (1981) "O Superman", One Ten Records, OTR005.

Armstrong, Louis and his Hot Fives (1925) "Yes, I'm In The Barrel", Okeh, Okeh8261-B.

Basie, Count (1937) "One O' Clock Jump", Decca, 62332.

Basie, Count (1937) "Jumpin' At the Woodside", Brunswick, A 81885.

Beatles, The (1967). Sgt. Pepper's Lonely Hearts Club Band, Capital, 4XAS 2653.

Beatles, The (1968). The White Album, Apple, SWBO-101.

Bodiansky, Dimitri and Varley, Nicolas (2002) "Syberia Theme" from game Syberia, Microids.

Broadcast (2006) "Chord Simple", The Future Crayon, Warp, WARPCD 146.

Bryars, Gavin (1986). "Sub Rosa", Vita Nova. ECM Records.

Chemical Brothers, The (2011). "Bahnhof Rumble". Hanna, Black Lot Music.

Chi-Lites, The (1974), "That's How Long", Brunswick, BR 15.

Cooke, Sam (1960), "Wonderful World", Keen Records, 2112.

Cranes (2004) "Particles and Waves", Particles and Waves, Dadaphonic, DADA 003.

Danger Mouse (2004), The Gray Album, Self Release, Gray LP1.

Eno, Brian, Fripp, Robert (1973), No Pussyfooting, Island, Help-16.

Foals, The (2008), Antidotes, Transgressive Records, TRANS071CD.

Frisell, Bill,1983, In Line, ECM, 1241.

Howard, Adina (1995) "Freak Like Me" on Do You Wanna Ride?, EastWest

Records, 0-66175.

Human League (1980) Travelogue, Virgin, V 2160.

Killing Joke (1980) "Requiem" on Killing Joke, EG, EGMD 5.45.

King Crimson (1975) USA, Island, ILPS 9316.

LaFosse, Andre (2003), Normalized, Altruistic Music, 634479253553.

Lali Puna (2004) "Faking the Books", Faking the Books, Morr Music, MM044.

Lewis, Leona (2007), Spirit, Syco Music, 88697185262.

Muthspiel, Wolfgang, Blade, Brian (2008), Friendly Travellers Live, Material Records, MRE 017-2.

Presley, Elvis (1957) "All Shook Up", RCA, RCA 47-6870.

Presley, Elvis (1956) "Hound Dog", RCA, 20-6604.

Riley, Terry (1968) In C, CBS, S CBS 61237.

Oldfield, Mike (1973) Tubular Bells, Virgin, V2001.

The Kinks (1964), "You Really Got Me", Pie, 7N 15673.

Riley, Terry (1966) Reed Streams, Mass Art Inc, M-131.

Riley, Terry (1969) Rainbow in Curved Air, CBS Masterworks, MS 7315.

Schaeffer, Pierre (1948) "Etudes aux Chemin de Fer", Cinq Etude de Bruits, (radio broadcast, Paris).

Son of Dave (2003) 02, Husky Records · HR006.9.

Stereolab (1993) "Lock Groove Lullaby", Transient Random Noise Bursts With Announcements, Elektra, 61536-2. 
The Rolling Stones (1965) "(I Can't Get No) Satisfaction", Decca, F12220.

Sugababes (2002) "Freak Like Me", Angels With Dirty Faces, Island, CID8122.

Super Furry Animals (2001) Rings Around the World, Epic, 5024132.

Tubeway Army (1979) "Are 'Friends' Electric?", Replicas, Beggars Banquet, BEGA/C 7.

Tunng (2004) "Tale From Black", Mother's Daughers and Other Songs. Static Caravan VAN72.

Z, Jay (2003) The Black Album, Roc-A-Fella-Records B0001528-02.

Interviews

Andre LaFosse, in email correspondence with the authors. October 21, 2011 